\title{
Ribosome biochemistry in crystal structure determination
}

\author{
GULNARA YUSUPOVA and MARAT YUSUPOV \\ Biologie Structurale Intégrative, Institut de Génétique et de Biologie Moléculaire et Cellulaire, Université de Strasbourg; CNRS, UMR7104; \\ INSERM, U964, Illkirch, 67400, France
}

Protein synthesis is carried out by ribosomes, the universal ribonucleoprotein assemblies, where genetic information encoded in messenger RNA is translated into a polypeptide chain. These giant organelles, which have a molecular weight of $2.5 \mathrm{MDa}$ in bacteria and up to $4.5 \mathrm{MDa}$ in higher organisms, are composed of many different proteins and long RNA chains. Although the ribosome and its basic functions were discovered more than 50 years ago, the large size (approximately 4600 nucleotides and 55 proteins) and highly dynamic nature of this macromolecule made it difficult to obtain ribosomes in crystal form. The first X-ray structures of whole bacterial ribosome, $30 \mathrm{~S}$ bacterial, and $50 \mathrm{~S}$ archaea subunits triggered an avalanche of new works in the ribosome field, which vastly increased our understanding of the functioning of this molecular machine.

For decades, it has been clear that there is no superior method for understanding a machine as complicated as the ribosome to capture an atomic-scale picture. Only one technique could conceivably provide the high-resolution structural information that the ribosome field ultimately required: X-ray crystallography. However, the relevance of this technique was uncertain for a long time. Although many protein and several nucleic acid structures had been solved by the 1980s, X-ray crystallography applied to an asymmetric macromolecule such as the ribosome was beyond consideration. Macromolecules (2.5 Mega Dalton and more) must be crystallized before their structures can be solved by X-ray crystallography, and for many years, no 70S ribosome crystals with diffracting abilities existed.

It is important to underline that the recent progress in ribosome crystallization for X-ray structure determination was based on the many previous biochemical and hydrodynamic studies of individual ribosomal components in solution, such as ribosomal RNA, individual ribosomal proteins, ribosomal subunits and ribosome functional complexes, which were accumulated over many decades.

Here we summarize the key steps in the biochemical study of ribosomes, focusing primarily on the work from our laboratory, which led to the first crystal structure elucidation of

\footnotetext{
Corresponding author: marat.yusupov@igbmc.fr

Article and publication date are at http://www.rnajournal.org/cgi/doi/10. 1261/rna.050039.115. Freely available online through the RNA Open Access option.
}

prokaryotic $70 S$ ribosomal complexes, and our recent progress in elucidating the first crystal structure of the complete 80 S eukaryotic ribosome.

The first visualization of the shape of the bacterial ribosome and its non-symmetric ribosomal subunits was obtained by the reconstitution of negatively stained electron microscopy (EM) images. Then, cryo-EM and single-particle analysis produced the first direct visualizations of the bacterial ribosome in different functional states. However, when the X-ray crystallographic structures of the entire 70S ribosome (as well as those of the individual $30 \mathrm{~S}$ and $50 \mathrm{~S}$ subunits) emerged, accurate atomic models became available.

In the 1980s, the first efforts to obtain usable three-dimensional crystals of ribosomal subunits were conducted by Ada Yonath's group in collaboration with Gunter Witmann at the Max-Planck Institute in Berlin. Yonath developed crystallization methods for $50 \mathrm{~S}$ ribosomal subunits isolated from Bacillus stearothermophilus and Haloarcula marismortui. Her pioneering work provided the crystals of large prokaryotic and archaea 50S subunits diffracting to $3.0 \AA$ resolution.

At that time, our group was working at the Institute of Protein Research at the Academy of Sciences (USSR) in Pushchino, which is primarily interested in the biochemistry of nucleic acids and protein biosynthesis. Our ribosome crystallography project was initiated in 1986 as collaboration between the Institute of Protein Research and Institute of Crystallography in Moscow. This agreement was between Alexander Spirin and Boris Vainshtein. The primary goal of this collaboration was the development of a new method for the purification and crystallization of more stable ribosomes from organisms that grow under extreme conditions, which may resist crystal growth conditions. Thus, the bacterium Thermus thermophilus, an extreme thermophile with an optimal living temperature $75^{\circ} \mathrm{C}$, was introduced into ribosome crystallography and continues to be a useful model for current translational crystallographic studies.

High-density sucrose- $\mathrm{CsCl}$ is the first step of purification, and Toyopearl-Butyl hydrophobic chromatography of ribosomal tight couples renders ribosomes suitable for crystallization. This basic protocol, with minor modifications, was used

(C) 2015 Yusupova and Yusupov This article, published in RNA, is available under a Creative Commons License (Attribution-NonCommercial 4.0 International), as described at http://creativecommons.org/licenses/by-nc/4.0/. 
for the crystallization of three ribosome crystal types. The first crystal form was obtained at the Institute of Protein Research and confirmed the principal possibility of using X-ray to study the whole ribosome and its functional complexes. The second crystal form was used for the first structural determination of a ribosome containing mRNA and tRNA at $7.8 \AA$ resolution and then at $5.5 \AA$ resolution. This study was conducted in collaboration with Harry Noller at the Center for Molecular Biology of RNA at the University of California at Santa Cruz. The third crystal form was obtained in the laboratory of Venki Ramakrishnan and used for the structural determination of the ribosome at $2.8 \AA$ resolution. The structure of the ribosome at $3.5 \AA$ resolution from Escherichia coli was determined previously in the laboratory of Jamie Cate.

Our group also obtained the first crystals of the $30 \mathrm{~S}$ ribosomal subunit from T. thermophilus. After optimization of crystal growth and $\mathrm{X}$-ray data collection conditions, this crystal form was used for the structural determination of the first 30 S subunit by laboratories of Venki Ramakrishnan and Ada Yonath. After optimization of crystal growth conditions and $\mathrm{X}$-ray data collection conditions, the crystal form obtained by Ada Yonath laboratory for the 50S ribosomal subunit from $H$. marismortui was used for X-ray structure determination by the laboratories of Peter Moore and Tomas Steitz. These crystals of individual ribosomal subunits have been used to study complexes with mimics of functional ligands as mRNA and tRNA. High-resolution structures of the $T$. thermophilus $30 \mathrm{~S}$ subunit and $H$. marismortui $50 \mathrm{~S}$ subunit and experimental electron density maps of the full $70 \mathrm{~S}$ ribosome containing three tRNAs and mRNA have been used by our group for modeling the full ribosome of T. thermophilus.

Over the last decade, remarkable advances have been made in full ribosome crystallography, to the extent that it is now possible to obtain a medium- or high-resolution structure of $70 \mathrm{~S}$ ribosome complexes with key components, such as mRNA and tRNAs. These structural studies have helped to create new complexes and explain the mechanism of tRNA binding in the presence of the elongation factor $\mathrm{Tu}$, the processes of mRNA decoding and peptide-bond formation and the mechanism of GTP hydrolysis, as well as translocation, termination and ribosomal recycling. Crystallography of full ribosome complexes has also been used for co-translational modification studies and translational regulation.

This wealth of information gained from high-resolution crystal structures of prokaryotic ribosomes enhanced our knowledge of prokaryotic protein synthesis; however, until 2010, our understanding of the eukaryotic ribosome was limited, as efforts to elucidate the structure of the eukaryotic ribosome remained unsuccessful. Despite the evolutionary conservation of ribosomal RNA (rRNA) and ribosomal proteins, there are substantial differences between prokaryotic and eukaryotic ribosomes. Due to the presence of $\sim 30$ additional proteins and more than 50 additional nucleotide sequences, called RNA expansion segments, that are inserted into the evolutionary conserved rRNA core, eukaryotic ribosomes have an $\sim 40 \%$ larger mass than prokaryotic ribosomes. Prior to 2010, knowledge of the structural organization of eukaryotic ribosomes was based on fitting highresolution X-ray structures of prokaryotic ribosomes into medium-resolution cryo-EM single-particle reconstructions.

The first indication that the eukaryotic ribosome could be crystallized was provided in 1966, when two-dimensional ribosome crystals were discovered in the tissues of hypothermic chick embryos. However, efforts to crystallize eukaryotic ribosomes were unsuccessful until recently, despite the highly competitive field of structural biology. These difficulties likely stem from the larger size (minimal molecular weight of 3.3 $\mathrm{MDa}$ in yeast and plants) and more complex biochemistry, as mentioned previously.

However, in 2010, we succeeded in crystallizing the whole eukaryotic 80 S ribosome from Saccharomyces cerevisiae and obtained the first X-ray structure of eukaryotic ribosome initially at $4.15 \AA$ resolution and then at $3.0 \AA$ resolution.

The successful crystallization of the ribosome isolated from yeast was again based on the development of a new method for the purification of intact eukaryotic ribosomes. First, we utilized the observation that glucose starvation of growing yeast cells inhibits the initiation and accumulation of very homogenous ribosomes without any ligands. Thus, cells for ribosome purification pass through the glucose starvation step to increase the initial quantity of ribosomal monomers. Second, a very gentle isolation protocol was developed to ensure that all of the ribosomal components were intact and present. Adam Ben-Shem introduced mild fractionation of S30 cellular extract by polyethylene glycol 20,000 with subsequent purification of $80 \mathrm{~S}$ ribosomes on a sucrose gradient under non-dissociation conditions. Thus, a very homogenous sample for crystallization was isolated, which yielded well-diffracting crystals.

The crystal structures of the eukaryotic ribosome from Saccharomyces cerevisiae significantly increased the understanding of protein synthesis and its regulation in the cell. For example, an analysis of the crystal structure of the yeast ribosome purified from cells subjected to glucose starvation revealed that the non-ribosomal protein Stm1 bound to the $80 \mathrm{~S}$ ribosome. This protein creates an additional bridge between two ribosomal subunits and increases the stability of the ribosome in crystallization conditions. The $80 \mathrm{~S}$ ribosome complexes with eukaryote-specific or broad-spectrum inhibitors not only enabled researchers to define common principles of targeting and resistance but also enabled improvements in the quality of crystals and collection of Xray data at $2.8 \AA$ resolution.

The $40 \mathrm{~S}$ and $60 \mathrm{~S}$ ribosomal subunits from the eukaryotic organism Tetrahymena thermophila were successfully crystallized with protein factors in the laboratory of Nenad Ban, and the complex structures were determined at $3.8 \AA$ and $3.6 \AA$ resolution, respectively.

The significant biochemical and hydrodynamic background of the ribosome field allowed us to transform this 
knowledge into the crystallization and X-ray structural interpretation of bacterial and then yeast ribosomes. The first crystal structure of the $80 \mathrm{~S}$ eukaryotic ribosome represents a breakthrough in structural/functional studies of eukaryotic ribosomes and enables the rationalization, in structural terms, of existing biochemical and genetic information, eventually facilitating the design of future experimental models for investigating various aspects of protein synthesis.

Unfortunately, the ribosome biochemistry of highly developed eukaryotes, including human ribosomes and ribosomes of the mitochondria, is not well elucidated and will require additional biochemical and hydrodynamic studies to obtain suitable crystals for X-ray analysis and other structural biology methods. For example, human ribosome has a molecular weight 4.5 MDalton, which is 1 MDalton more than the yeast ribosome. This difference is due to expansion segments of the ribosomal RNA of the 60S subunit. Cryo-EM analysis has demonstrated that these expansion segments are not well defined in the human ribosome structure.
Cryo-EM analysis also revealed that mitochondrial ribosomes are not sufficiently compact to enable a complete interpretation of the structure. These studies demonstrated a well-defined structure of the large ribosomal subunit; however, the small subunit was flexible and not able to be interpreted. Additionally, very little is known about the ribosomes of Gram-positive bacteria and many pathogenic organisms. These studies would increase our knowledge of the mechanism of protein synthesis and regulation in these organisms and will help in developing new drugs and antibiotics.

\section{Acknowledgments}

Our own work summarized in this paper was supported by the French National Research Agency ANR-11-BSV8-006 01 (to G.Y.), the European Research Council advanced grant 294312 (to M.Y.), the Human Frontier Science Program grant RGP0062/2012 (to M.Y.), the Russian Government Program of Competitive Growth of Kazan Federal University (to G.Y. and M.Y). 

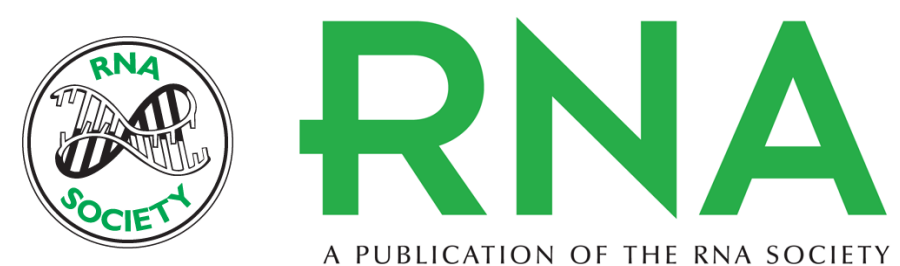

A PUBLICATION OF THE RNA SOCIETY

\section{Ribosome biochemistry in crystal structure determination}

Gulnara Yusupova and Marat Yusupov

RNA 2015 21: 771-773

Open Access Freely available online through the RNA Open Access option.

Creative This article, published in RNA, is available under a Creative Commons License

Commons (Attribution-NonCommercial 4.0 International), as described at

License http://creativecommons.org/licenses/by-nc/4.0/.

Email Alerting Receive free email alerts when new articles cite this article - sign up in the box at the Service top right corner of the article or click here. 